\title{
Charging of gold/metal oxide/gold nanocapacitors in a scanning electron microscope
}

\author{
Michael J Coutts ${ }^{1}$, Hadi M Zareie ${ }^{2,3}$, Michael B Cortie ${ }^{1}$ and \\ Andrew M McDonagh ${ }^{1}$ \\ ${ }^{1}$ Institute for Nanoscale Technology, University of Technology Sydney, PO Box 123, \\ Broadway NSW 2007, Australia \\ ${ }^{2}$ Department of Material Science and Engineering, Izmir Institute of Technology, Gulbahce, Urla, \\ 351430, Izmir, Turkey \\ ${ }^{3}$ Microstructural Analysis Unit, School of Physics and Advanced Materials, University of Technology \\ Sydney, PO Box 123, Broadway NSW 2007, Australia \\ E-mail: Andrew.McDonagh@uts.edu.au
}

Received 14 November 2013, revised 29 January 2014

Accepted for publication 19 February 2014

Published 20 March 2014

\begin{abstract}
Triangular parallel-plate nanocapacitors were fabricated by a combination of microsphere lithography and physical vapor deposition. The devices were comprised of a $20 \mathrm{~nm}$ layer of dielectric material sandwiched between two $20 \mathrm{~nm}$ layers of gold. Dielectric materials with a range of relative permittivities were investigated. Charging of the capacitors was probed in a scanning electron microscope (SEM) by monitoring the change in brightness of the images of the devices as a function of time. The time constants, $R C$, associated with the charging of the capacitors, were extracted from the SEM grayscale data. The resulting average $R C$ values were $248 \pm 27 \mathrm{~s}$ for $\mathrm{SiO}_{2}, 70 \pm 8 \mathrm{~s}$ for $\mathrm{Al}_{2} \mathrm{O}_{3}, 113 \pm 80 \mathrm{~s}$ for $\mathrm{ZnO}$ and $125 \pm 13 \mathrm{~s}$ for $\mathrm{HfO}_{2}$. These values are consistent with the anticipated $R C$ values based on the resistivities and permittivities of the materials used in the devices and importantly, were measured without the need to attach any wires or leads.
\end{abstract}

Keywords: nanosphere lithography, nanocapacitors, charging, scanning electron microscopy

S Online supplementary data available from stacks.iop.org/Nano/25/155703/mmedia

(Some figures may appear in colour only in the online journal)

\section{Introduction}

There is significant interest in developing methods to fabricate macroscale capacitors comprised of nanoscale materials [1-3]. Popular approaches include synthesizing electrochemical capacitors that incorporate carbon nanotubes [4] or graphene sheets [5] in electrodes. Functionalization [6, 7], or incorporation of composite materials $[8,9]$ for synergistic effects (such as improved power and energy densities) are also frequently investigated. Somewhat less attention has been paid to the soft lithographic fabrication of discrete nano-or mesoscale capacitors but, with the continuing miniaturization of other circuit components, such tiny capacitors may become invaluable for future electronic devices.

Traditional MOS (metal/oxide/semiconductor) or MOM (metal/oxide/metal) capacitors are commonly characterized by capacitance-voltage measurements where a small AC voltage is applied to the device while measuring the current. The current can then be integrated with respect to time to determine the charge, and hence the capacitance. Continued miniaturization of capacitance devices makes this type of measurement increasingly difficult because (1) the parasitic capacitance of any attached leads may eventually mask the measurement and (2) it may be challenging to attach 


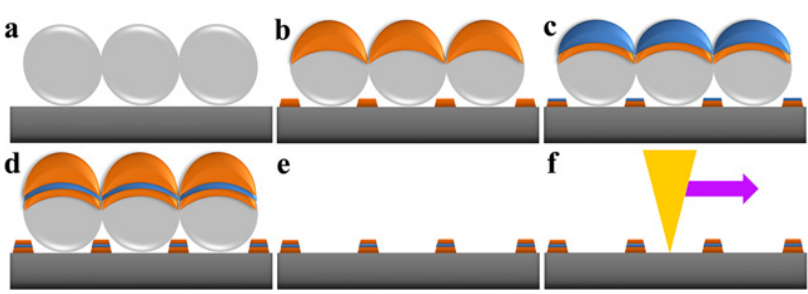

Figure 1. Schematic representation of the fabrication of discrete nanoscale capacitors and the charging process. (a) Close-packed latex spheres on silicon prepared by spin-coating. (b) Close-packed spheres act as a mask so that gold deposited by evaporative deposition forms discrete, patterned structures on the silicon substrate. Gold also deposits on the latex spheres. (c) A metal oxide layer is deposited by RF magnetron sputtering. (d) A second layer of gold is deposited by evaporative deposition. (e) Removal of the latex spheres results in periodic nanostructured arrays of capacitive triangles. (f) Charging of the nanostructures is achieved by repeatedly scanning an electron beam (represented by yellow triangle) across the sample.

such leads anyway. This is particularly the case for discrete nanocapacitors produced by self-assembly or soft lithography. Therefore, alternative methods to characterize the smallest capacitors are needed, especially those produced by nontraditional routes. Monolayer-protected clusters and metal carbonyl clusters have been characterized by cyclic voltammetry or differential pulse voltammetry [10], while the pioneering $\mathrm{Au} @ \mathrm{SnO}_{2}$ colloidal nanocapacitors of Mulvaney et al [11] were probed by changes to the optical properties. However, these techniques probe particle ensembles rather than individual particles. Scanning tunneling or conductive atomic force microscopy can be used to probe the charging of individual nanodevices [12], but only within a relatively narrow window of voltages and timescale.

Scanning electron microscopy (SEM) is a versatile, powerful technique that has become indispensable in fields such as nanotechnology and materials science. The injection and emission of high energy electrons into samples allows information such as surface topography, sample composition and electrical conductivity to be extracted. In preliminary work, our group has demonstrated that SEM can also be used to experimentally estimate the capacitance of discrete nanocapacitors [13]. The basis of the capacitance measurement technique involves imaging arrays of discrete nanocapacitors at low accelerating voltages, and monitoring the increase in brightness of these devices over time. The increase in brightness is a consequence of an increasingly greater emission of secondary electrons and hence of an increasingly negative surface potential [14]. This change in brightness enables the time constant for charging to be measured and, consequently, the capacitance to be estimated.

Here we investigate the use of this relatively new measurement technique to characterize the effect of materials of construction on the charging of discrete nanocapacitors produced by nanosphere lithography. These structures consist of a metal oxide layer 'sandwiched' between two layers of gold. Architectures using gold/silicon oxide 'nanosandwiches' have been shown previously to exhibit interesting optical responses [15] but here we are concerned with how behavior
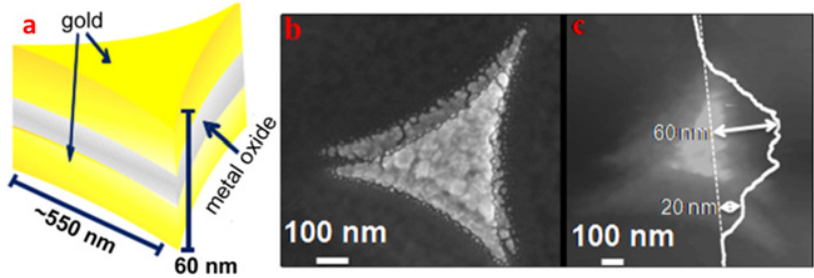

Figure 2. (a) Schematic representing the nanostructures produced for charging experiments. (b) Scanning electron micrograph of a nanostructure fabricated by depositing $20 \mathrm{~nm}$ of gold $/ 20 \mathrm{~nm}$ of alumina/20 nm of gold (deposition of top layer of gold was offset in this example by tilting the sample during the last deposition cycle in order to facilitate visualization of the uppermost gold layer). Image parameters: accelerating voltage, $20 \mathrm{kV}$, working distance, $1.8 \mathrm{~mm}$. (c) AFM image and section line profile of $20 \mathrm{~nm}$ gold $/ 20 \mathrm{~nm}$ hafnia/20 $\mathrm{nm}$ gold nanostructure (structure synthesized with a larger offset of bottom gold layer to aid film thickness measurements).

in the SEM can be exploited to extract the capacitors' $R C$ constant. The technique provides a lead-free way to characterize a key electrical property (the $R C$ constant) of discrete nanoscale structures, however, as we will show, a limitation is that determination of the capacitance itself requires an estimate of the resistivity of the actual thin film, which may not be readily available.

\section{Results and discussion}

Figure 1 depicts the fabrication and charging processes for the nanocapacitors prepared in the current work. Monodisperse polystyrene latex spheres were deposited on piranhaetched silicon substrates by spin-coating, figure 1(a). The spheres form a hexagonal close-packed structure that acts as a mask [13, 15-20] for the subsequent multilayer deposition of gold/metal oxide/gold films, figures 1(b)-(d). In this work, a number of different metal oxide dielectrics were investigated including alumina $\left(\mathrm{Al}_{2} \mathrm{O}_{3}\right)$, zinc oxide $(\mathrm{ZnO})$, silica $\left(\mathrm{SiO}_{2}\right)$, hafnia $\left(\mathrm{HfO}_{2}\right)$ and titania $\left(\mathrm{TiO}_{2}\right)$. Removal of the spheres, figure 1(e), results in periodic arrays of gold/metal oxide/gold nanocapacitors. Figure 1(f) depicts the charging of the nanocapacitors using a focused electron beam in a scanning electron microscope.

The geometry of the fabricated nanostructures is shown in figure 2(a) together with the nanostructure dimensions. Figure 2(b) shows a scanning electron micrograph of a gold/alumina/gold structure in which the top gold layer is offset to illustrate the sandwich-type structure (the alumina layer in this image is not visible due to low contrast under the chosen imaging conditions). Atomic force microscope measurements, figure 2(c), revealed that the overall height of the nanostructures is $60 \mathrm{~nm}$ (the sum of the three $20 \mathrm{~nm}$ thick films).

Figure 3 shows SEM images that have been acquired sequentially of the same area of $\mathrm{Au} / \mathrm{Al}_{2} \mathrm{O}_{3} / \mathrm{Au}$ nanostructures. The accelerating voltage of $0.3 \mathrm{kV}$ used for imaging is critical to the experiments described here. Monte Carlo simulations [13] have shown that, for gold, the radius of the electron beam interaction volume is $\sim 3 \mathrm{~nm}$ using this accelerating voltage. At this voltage, the initial induced surface potential should be close to zero because the accelerating 


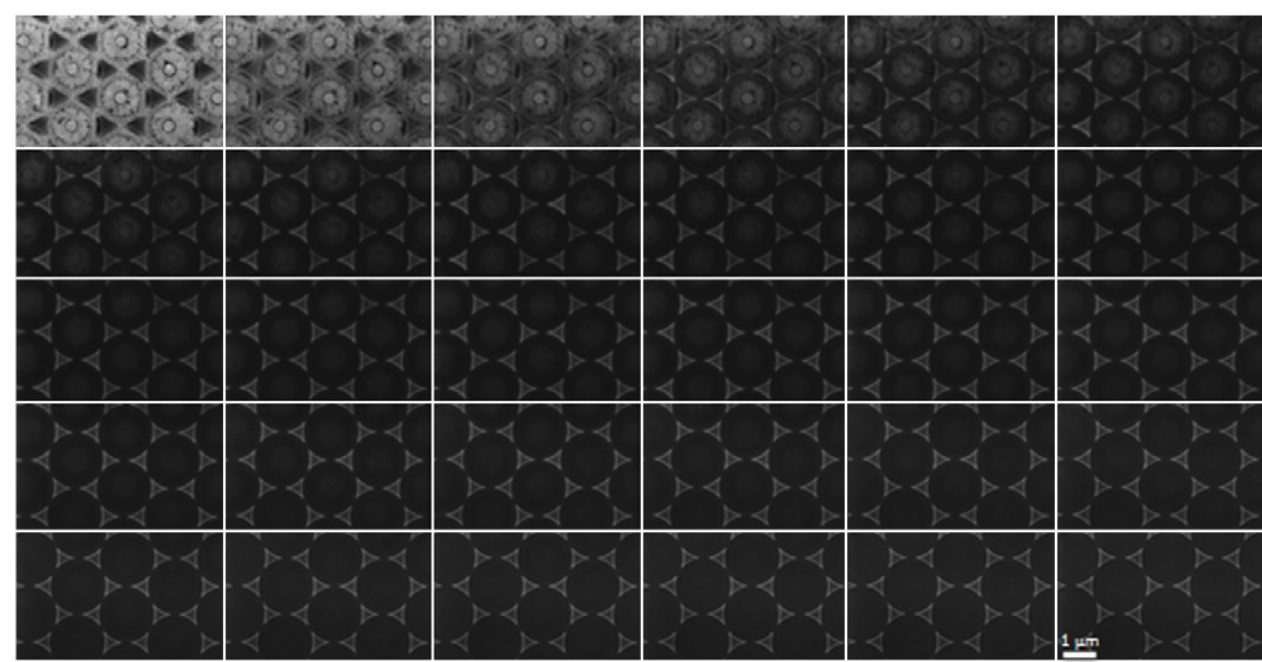

Figure 3. SEM micrographs of a gold $(20 \mathrm{~nm}) /$ alumina $(20 \mathrm{~nm}) /$ gold $(20 \mathrm{~nm})$ nanostructure array collected over time (timescale: $\sim 45 \mathrm{~s}$ /image). Individual images are ordered left to right, top to bottom (additional images are available in the supplementary information available at stacks.iop.org/Nano/25/155703/mmedia). The circular structures visible in the first row of images are assigned to a small amount of residual organic material arising from the detachment process of the polystyrene spheres.

voltage is very close to the first critical energy $\left(E_{\mathrm{c} 1} \approx 0.3 \mathrm{keV}\right)$ of gold [14, 21]. In addition, at such low beam potentials the electron beam penetrates only the topmost layer of the layered structure. As electrons flow into the electrically floating $\mathrm{Au}$ top electrode, the surface potential will become negative as the capacitor charges.

Figure 3 reveals that the initially dark triangular nanostructures increase in brightness upon continuous imaging due to an accumulation of electrons in the topmost gold layer. Over the same period, the silicon substrate becomes darker. The mechanism of darkening on the silicon involves a build-up of positive charge on the silicon surface, which has the effect of retarding the escape of secondary electrons [14]. The positive charge arises due to the need to balance the floating negative charge on the uppermost gold electrode. The evolution of mean grayscale intensity for each nanocapacitor was measured as well as that of their silicon substrates to quantify the dynamics of the deposition and charging processes. We emphasize that grayscale intensity is not an arbitrary property, but rather quantitative data where each pixel in an SEM image is assigned a single value of intensity from black, which is assigned a value of 0 , through to white, 256. The grayscale intensity correlates with charging whereby higher intensity values (equating to brighter regions) indicate greater negative surface charge densities.

Figure 4 shows a graph of the mean grayscale intensity versus imaging time for each of the triangular $\mathrm{Au} / \mathrm{Al}_{2} \mathrm{O}_{3} / \mathrm{Au}$ nanostructures shown in figure 3 together with that of the nearby silicon substrate. These data provide insight into the charging profiles for individual devices. The mean intensities vary somewhat for individual structures, which reflects the effect of small changes in structure geometries that can be attributed to inter-sphere void size differences in the masking phase of fabrication. The structures undergo three relatively distinct brightness changes during continuous imaging. Between 0 and $\sim 450 \mathrm{~s}$, region 1 , the intensity varies somewhat erratically for each structure but in general diminishes

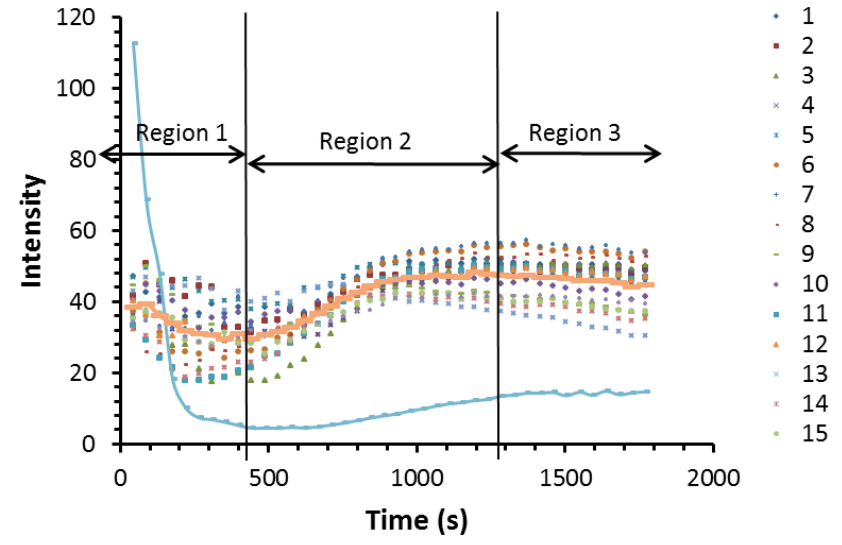

Figure 4. Graph showing mean grayscale intensities for fifteen gold/alumina/gold nanodevices as a function of time. Also shown is the mean grayscale intensity of the entire silicon substrate (blue trace) which surrounds the nanodevices.

somewhat. Between $\sim 450$ and 1200 s, region 2 , the intensity increases with an initially high, but slowing rate. After $1200 \mathrm{~s}$, region 3, the intensity remains relatively constant although for some structures it decreases marginally with time. The gold/dielectric interface is not directly probed in these experiments but rather only the uppermost few nanometers of the various surfaces. Therefore, in region 1 the triangles darken due to the applied potential of the electron beam resulting in a charge build-up at the gold/dielectric interface, attracted there to compensate the growing positive charge in the substrate. This results in fewer electrons at the surface of the triangles (figure 5(a)). In region 2, brightening of the electrically isolated metallic parts is associated with an increase in negative potential of the surface of the gold top electrode. This we associate with a saturated dielectric and thus an increasing electron density at the surface as more electrons are injected from the beam. Additional electrons from the beam also cause a slow reduction in the net positive charge of the 

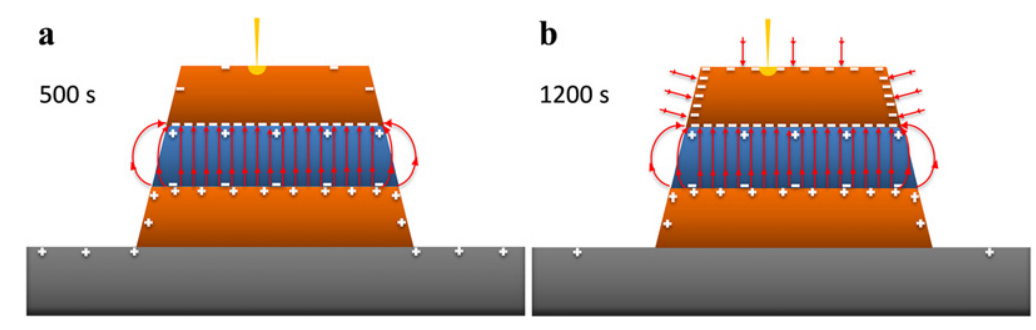

Figure 5. Schematic representation of the charge configuration of a single $\mathrm{Au} / \mathrm{Al}_{2} \mathrm{O}_{3} / \mathrm{Au}$ nanostructure at (a) $t \sim 500 \mathrm{~s}$ and (b) $t \sim 1200 \mathrm{~s}$ Note that the substrate becomes less positively charged after $500 \mathrm{~s}$ due to the continuing incoming current of electrons from the beam which can only escape by leaking slowly to ground.

Table 1. Calculated and experimental time constants and associated data.

\begin{tabular}{lllll}
\hline Material & Relative permittivity $\left(\varepsilon_{\mathrm{r}}\right)$ & Resistivity $(\Omega \mathrm{m})$ & Estimated time constant $(\mathrm{s})^{\mathrm{a}}$ & Experimental time constant $(\mathrm{s})$ \\
\hline Titanium dioxide & $86[22]$ & $\sim 10[22]$ & $7.6 \times 10^{-9}$ & $\mathrm{~b}$ \\
Zinc oxide & $7.5-8.6[23]$ & $\sim 10^{12}[24]$ & $70-80$ & $113 \pm 80$ \\
Alumina & $9.34[25]$ & $\sim 10^{12}[26]$ & 80 & $70 \pm 8$ \\
Silica & $4.42[27]$ & $\sim 10^{13}[28]$ & 390 & $248 \pm 27$ \\
Hafnia & $25-50[29,30]$ & $10^{8}-10^{11}[30,31]$ & $0.02-40$ & $125 \pm 13$ \\
\hline
\end{tabular}

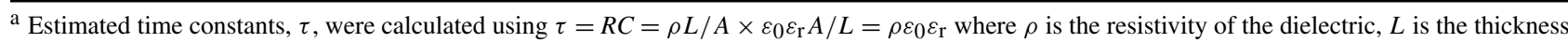
and $A$ is the cross sectional area of the device, $\varepsilon_{0}$ is the permittivity of free space and $\varepsilon_{\mathrm{r}}$ is the relative permittivity of the dielectric (or the material's dielectric constant).

$\mathrm{b}$ Too small for measurement using this technique.

substrate and hence a small increase in the substrate brightness is observed in this region. From $\sim 1200$ s onwards, region 3, the devices are fully charged throughout (figure 5(b)) and little further change occurs (i.e. a steady state of electron flux in and out of the uppermost triangle).

For the purpose of determining the charging rates of the capacitors, changes in the brightness of the gold surfaces do not provide a direct probe of charging at the gold/metal oxide interface since there is a period before the charge on the upper surface starts to become appreciably negative (region 1). On the other hand, the change in brightness of the silicon surfaces provides immediate information about the charging of the interface without the complications associated with the gold surface. Thus, the change in the grayscale intensity of the silicon surfaces for each of the device arrays during the charging phase (region 1) was used to measure the time constants of the devices. The charging of a capacitor obeys a first-order rate law and can be modeled by

$$
V(t)=V_{\max }\left(1-\mathrm{e}^{\frac{t}{\tau}}\right)
$$

where the time constant $(\tau)$ represents the time for a device to charge/discharge to $63.2 \%$ of the difference between its initial and final values, voltage $V(t)=0$ at $t=0, V(t)=V_{\max }$ at very long times, and

$$
\tau=R C
$$

where $R$ is the resistance and $C$ is the capacitance of the device. Experimental time constants were extracted from plots of grayscale intensities of the silicon substrates versus time $(t)$ fitted using $I(t)=I_{\infty}+A \mathrm{e}^{\frac{-t}{R C}}$, where $I(t)$ is the grayscale intensity at time $t, I_{\infty}$ is the minimum grayscale intensity in region 1.

Table 1 shows the time constants obtained from our measurements as well as estimated time constants based upon literature data for comparison. We emphasize that the estimated $R C$ data is useful as a guide only as such data is quite sensitive to individual deposition conditions. This factor also implies that the literature data for the material properties must be taken as only a first-order approximation for the purposes of the present work. Ideally, the actual resistivity and permittivity of the thin films should be measured if performance-critical estimates of capacitance are required. Devices that contain $\mathrm{TiO}_{2}$ as the dielectric material did not exhibit any measurable charging behavior. This is as expected as films of titanium dioxide with thickness $<50 \mathrm{~nm}$ have been shown to be reasonably conductive [22]. Zinc oxide, silica, alumina, and hafnia show theoretical time constants that are in the region of tens to hundreds of seconds and are quite amenable to measurement with our experimental conditions. The experimental time constants of the devices containing zinc oxide, silica and alumina are all in agreement with those predicted based upon the relative permittivities and resistivities of these materials while the experimental time constant for hafnia-containing devices is somewhat greater than that predicted. In studies involving thin films of metals and metal oxides, the phenomenon of defect formation, in particular at the oxide surface, may play a role in the electronic properties of the subsequent assemblies and such phenomena would provide an interesting focus for further investigation with regard to nanoscale capacitor devices.

A prolonged charging experiment was conducted with $20 \mathrm{~nm}$ gold/20 nm silica/20 nm gold nanostructures, which 


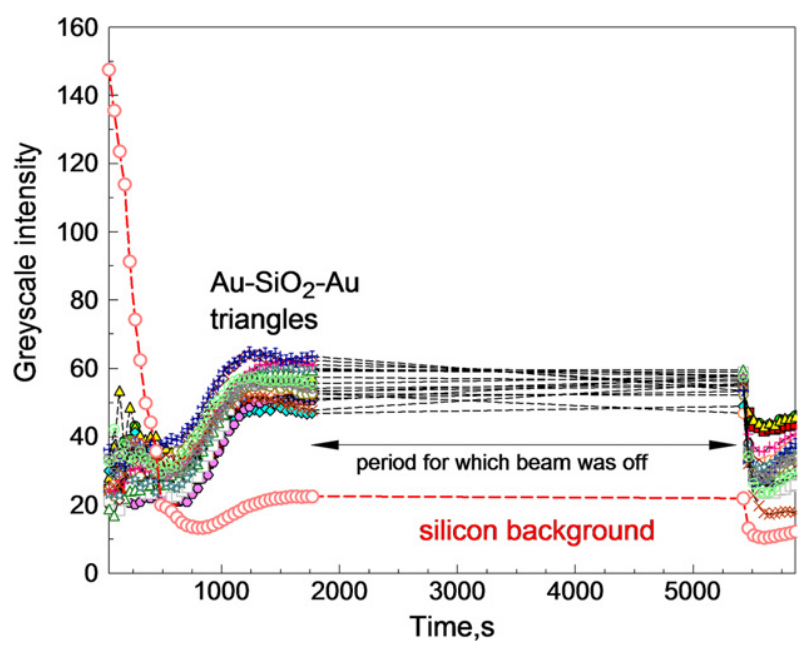

Figure 6. Plot of mean gray scale intensity for $\mathrm{Au}-\mathrm{SiO}_{2}-\mathrm{Au}$ triangular structures, showing effect of blanking the electron beam for $1 \mathrm{~h}$. Mean silicon substrate intensity is also presented. No data where recorded during the blanked beam period (represented by dashed lines).

included an hour during which time the electron beam was blanked. The results are shown in figure 6. It is evident that the charged state of the capacitors was substantially maintained during the hour in which the electron beam was absent (the silicon substrate intensity does not return to its uncharged value). When the beam was blanked after charging the capacitors, excess charge (i.e., the charge applied by the beam after saturation of the dielectric, region 2) dissipated. The capacitors, however, remain charged and so upon re-introduction of the beam, behavior corresponding to the end of region 1 / beginning of region 2 (figure 4 ) is observed.

Throughout the charging experiments, SEM images of the edges of the individual nanostructures were significantly brighter than the central regions and, although both regions exhibited a brightness increase in the 500-1200 s period, the centers showed significantly less change and were less bright overall (figure 7). The edge effect is well known in scanning electron microscopy where a reduced escape distance results in a greater number of secondary electrons reaching the detector. However, this effect should only be visible at displacements of less than $3 \mathrm{~nm}$ from the edge (within the beam's interaction volume) [13]. Interestingly, analysis of figure 3 and other SEM images show that the edge is significantly brighter for displacements of up to $\sim 20 \mathrm{~nm}$.

Finally, we briefly discuss some control experiments. Gold-only triangles on silicon (see supplementary information available at stacks.iop.org/Nano/25/155703/mmedia) exhibited relatively small intensity changes upon scanning indicating very little capacitance as expected. The small $\tau$ is attributed to a very small $R$ caused by electron tunneling and leakage through the nanoscale native oxide film. In this case, charge on the top electrode drains relatively rapidly to ground.

A relatively minor degree of darkening of silicon was observed in the absence of any nanocapacitors. Such darkening is ubiquitous during SEM imaging in general and is due to the deposition of carbonaceous contaminants from the SEM chamber. To examine the influence of this darkening,

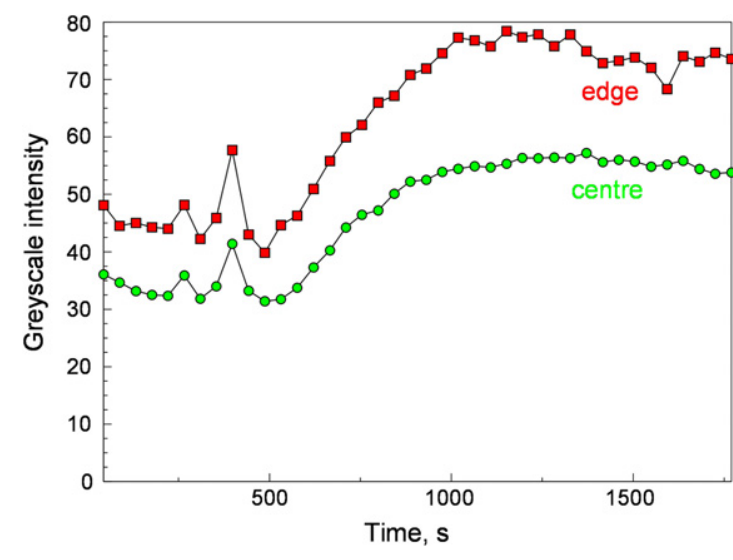

Figure 7. Graphs of mean gray scale intensities versus time for edge (red) and middle (blue) regions of a single $\mathrm{Au} / \mathrm{Al}_{2} \mathrm{O}_{3} / \mathrm{Au}$ structure ('Triangle \#7').

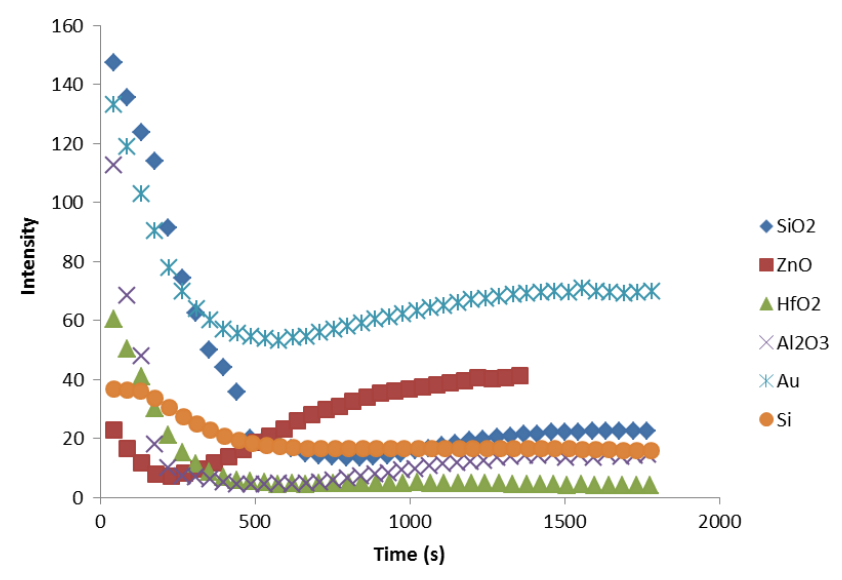

Figure 8. Graphs of grayscale intensity versus time for the silicon background for arrays of devices as well as unmodified silicon and gold-only arrays.

the intensity changes of the silicon background for each of the different arrays of nanostructures were compared, figure 8. Each of the metal-oxide-containing structures display the characteristic three-region behavior. The data also reveal that the rate of silicon darkening is, as expected, dependent upon the type of adjacent nanostructure and darkening due to in-chamber carbon deposition is quite small and evolves somewhat differently over time. It was also interesting to re-examine the comparator silicon substrate after three weeks of storage in ambient conditions. In this case, some charging is observed (supplementary information available at stacks .iop.org/Nano/25/155703/mmedia). We attribute this to the layer of native oxide becoming thick enough to retain some surface charge in the SEM. For capacitance measurements of fabricated devices such as those reported here, SEM experiments should be performed as close as possible to the completion of the deposition process.

\section{Experimental details}

\subsection{General}

The following materials were sourced commercially and used as received; silicon (p-type; 100, ProSciTech), sulfuric acid 
(Lab scan), hydrogen peroxide (33 wt\%) (Sigma-Aldrich), MilliQ water $\left(18.2 \mathrm{M} \Omega \mathrm{cm}^{-1}\right)$, latex spheres $(1.58 \mu \mathrm{m}$, $10 \mathrm{wt} \%$ solution, Bang Laboratories), Triton-X100 (Aldrich), methanol (Chem Supply), dichloromethane (Lab Scan), gold (AGR Matthey, fineness 999.9), zinc oxide (99.9\% Williams Advanced Materials), alumina (99.9\% Semiconductor Wafer Inc.), silica (Semiconductor Wafer Inc.), hafnia (99.9\% Semiconductor Wafer Inc.), and titania (99.9\% Williams Advanced Materials). Spin-coating was performed using a Headway Research spin-coater. Gold was deposited in a Denton Vacuum DV502 turbo evaporative deposition chamber. Metal oxides were deposited in an Edwards E306 deposition chamber equipped with a RF magnetron sputter system with an argon partial pressure of $3 \times 10^{-4}$ Torr. Deposition rate and film thickness were monitored with a Maxtek TM-200 quartz crystal film thickness monitor calibrated after deposition by AFM step height measurements.

\subsection{Nanosphere lithography}

Gold/metal oxide/gold arrays were fabricated using a modified literature procedure [22]. Silicon substrates (approx $\left.1 \mathrm{~cm}^{2}\right)$ were immersed in piranha solution $\left(3: 1 \mathrm{H}_{2} \mathrm{SO}_{4}: 33 \%\right.$ $\mathrm{H}_{2} \mathrm{O}_{2}$ CAUTION: this solution is a powerful oxidant and will destroy many organic materials) for $20 \mathrm{~min}$ and rinsed thoroughly with Milli- $Q$ water rendering the surface hydrophilic. A latex sphere solution $(400 \mu \mathrm{l})$ was added to $100 \mu \mathrm{l}$ of a solution of Triton-X100 in methanol (1:400 vol/vol). $20 \mu \mathrm{l}$ of the resultant suspension was dropped onto a silicon substrate and spun at $2000 \mathrm{rpm}$ for $40 \mathrm{~s}$. The substrate was then transferred to a vacuum deposition chamber equipped with a tungsten filament, $\sim 10^{-7}$ Torr $\left(\sim 10^{-5} \mathrm{~Pa}\right)$ base pressure. Gold was deposited by evaporative deposition with a deposition rate of $\sim 0.1 \AA \mathrm{s}^{-1}$. The distance between the sample and filament was $20 \mathrm{~cm}$. The substrate was then removed and transferred to a sputter equipped vacuum chamber and pumped to a base pressure $\sim 10^{-6}$ Torr $\left(\sim 10^{-4} \mathrm{~Pa}\right)$. Argon was leaked into the chamber to give a partial pressure of $3 \times 10^{-4}$ Torr $\left(4 \times 10^{-2} \mathrm{~Pa}\right)$, and metal oxides were deposited with an advanced energy RFX-600 power supply $(90 \mathrm{~W})$ under slow rotation $(\sim 0.2 \mathrm{~Hz})$. The distance between the sample and target was $15 \mathrm{~cm}$. Following metal oxide deposition, air was introduced into the chamber and the pressure equalized at atmospheric pressure. The gold evaporation procedure was then repeated. After the deposition process, the substrates were immersed in dichloromethane, sonicated for $2 \mathrm{~min}$, thoroughly rinsed with dichloromethane, and dried under a stream of nitrogen gas. No annealing was performed nor other procedures that might alter the structures of the nanodevices.

\subsection{AFM measurements}

Quartz crystal calibration measurements were performed on a Digital Instruments Dimension 3100 scanning probe microscope operating in tapping mode. Nanostructures were characterized on a Digital Instruments multimode scanning probe microscope in tapping mode.

\subsection{SEM experiments}

SEM measurements were obtained using a LEO Supra 55 VP microscope (Zeiss) equipped with an in-lens secondaryelectron detector. For charging experiments an accelerating voltage of $0.3 \mathrm{keV}$ was used and the scan rate was 44 s/image. Gray scale intensity measurements were obtained by selecting individual nanostructures or the entire silicon substrate from images and measuring the mean gray scale intensity with the histogram function of Adobe Photoshop giving the mean gray scale intensity for each selected area. When selecting the triangle structures, the 'lasso' function was used. To select substrate areas, the 'wand' tool was used with tolerances optimized for the particular background.

\subsection{Fitting of grayscale data}

The mean grayscale intensity of the silicon region of the arrays was used for estimating $\tau$. The data was fitted to the equation $I(t)=I(\infty)-A \mathrm{e}^{-t / R C}$ using FITYK 0.8.9 [32] to obtain estimates and confidence intervals for $R C$.

\section{Conclusions}

Nanocapacitors have been fabricated containing a range of dielectric materials. Nanosphere lithography coupled with RF magnetron sputtering reliably gave relatively large area arrays of devices. Estimates of the rate constant $\tau$ associated with the charging of the nanocapacitors were extracted by fitting grayscale data obtained using a scanning electron microscope. The $\tau(=R C)$ values were $248 \pm 27 \mathrm{~s}$ for $\mathrm{SiO}_{2}, 70 \pm 8 \mathrm{~s}$ for $\mathrm{Al}_{2} \mathrm{O}_{3}, 113 \pm 80 \mathrm{~s}$ for $\mathrm{ZnO}$, and $125 \pm 13 \mathrm{~s}$ for $\mathrm{HfO}_{2}$ The $R C$ values measured by this technique are consistent with those anticipated from the material properties of the dielectric interlayer.

Our methodology has proved useful for measuring time constants in the range of tens to hundreds of seconds. Values of $\tau$ were not extracted for devices containing $\mathrm{TiO}_{2}$ because the product of $R$ and $C$ for this material fell outside of the measurable range.

\section{Acknowledgments}

This work was supported by the Australian Research Council (DP0877539, DP0984354).

\section{References}

[1] Abruna H D, Kiya Y and Henderson J C 2008 Phys. Today 61 43-7

[2] Jayalakshmi M and Balasubramanian K 2008 Int. J. Electrochem. Sci. 3 1196-217

[3] Winter M and Brodd R J 2004 Chem. Rev. 104 4245-69

[4] An K H, Kim W S, Park Y S, Moon J M, Bae D J, Lim S C, Lee Y S and Lee Y H 2001 Adv. Funct. Mater. 11 387-92

[5] Peng X Y, Liu X X, Diamond D and Lau K T 2011 Carbon $493488-96$

[6] Hiraoka T, Izadi-Najafabadi A, Yamada T, Futaba D N, Yasuda S, Tanaike O, Hatori H, Yumura M, Iijima S and Hata K 2010 Adv. Funct. Mater. 20 422-8 
[7] Jeong H M, Lee J W, Shin W H, Choi Y J, Shin H J, Kang J K and Choi J W 2011 Nano Lett. 11 2472-7

[8] Gupta V and Miura N 2006 Electrochim. Acta 52 1721-6

[9] Yang S Y, Chang K H, Tien H W, Lee Y F, Li S M, Wang Y S, Wang J Y, Ma C C M and Hu C C 2011 J. Mater. Chem. 21 2374-80

[10] Hicks J F, Miles D T and Murray R W 2002 J. Am. Chem. Soc. 124 13322-8

[11] Oldfield G, Ung T and Mulvaney P 2000 Adv. Mater. 12 1519-22

[12] Cortie M B, Zareie M H, Ekanayake S R and Ford M J 2005 IEEE Trans. Nanotechnol. 4 406-14

[13] Zareie H M, Morgan S W, Moghaddam M, Maaroof A I, Cortie M B and Phillips M R 2008 ACS Nano 2 1615-9

[14] Cazaux J, Kim K H, Jbara O and Salace G 1991 J. Appl. Phys. 70 960-5

[15] Fredriksson H, Alaverdyan Y, Dmitriev A, Langhammer C, Sutherland D S, Zaech M and Kasemo B 2007 Adv. Mater. 194297

[16] Deckman H W and Dunsmuir J H 1983 J. Vac. Sci. Technol. B $11109-12$

[17] Haynes C L and Van Duyne R P 2001 J. Phys. Chem. B $1055599-611$

[18] Hulteen J C and Van Duyne R P 1995 J. Vac. Sci. Technol. A 13 1553-8
[19] Li C, Hong G S, Wang P W, Yu D P and Qi L M 2009 Chem. Mater. 21 891-7

[20] Pakizeh T, Abrishamian M S, Granpayeh N, Dmitriev A and Kall M 2006 Opt. Express 14 8240-6

[21] Seiler H 1983 J. Appl. Phys. 54 R1-R17

[22] Gu G R, He Z, Tao Y C, Lia Y A, Li J J, Yin H, Lia W Q and Zhao Y N 2003 Vacuum 70 17-20

[23] Ashkenov N et al 2003 J. Appl. Phys. 93 126-33

[24] Singh R, Kumar M and Chandra S 2007 J. Mater. Sci. 42 4675-83

[25] Young K F and Frederikse H P R 1973 J. Phys. Chem. Ref. Data 2 313-410

[26] Grffiths D 1999 Introduction to Electrodynamics 3rd edn (New Jersey: Prentice Hall)

[27] Demau C 1963 J. Phys. (Paris) 24 284-5

[28] Serway R A 1998 Principles of Physics (Fort Worth, TX: Saunders)

[29] Hsu C T, Su Y K and Yokoyama M 1992 Japan. J. Appl. Phys. 31 2501-4 (Part 1)

[30] Hsu C T, Li J W, Su Y K and Wu T S 1992 J. Appl. Phys. 71 1509-12

[31] Pereira L, Barquinha P, Fortunato E and Martins R 2005 Mater. Sci. Eng. B 118 210-3

[32] Wojdyr M 2010 J. Appl. Crystallogr. 43 1126-8 